\title{
Developing Machine Learning-based Mobile Application to Identify Ingredients of Korean Street Food
}

\author{
Rifqi Muhammad Fikri1), Mintae Hwang2)
}

\begin{abstract}
The tourist experience is highly affected by the local cuisine of a particular region. However, limited knowledge of local food can be challenging for tourists because they are mostly unfamiliar with the local language. This may increase the disliking tendencies toward a local culinary setting and impact the traveling experience. Therefore, in this study, we propose a mobile application to provide information about the local Korean street food ingredients. This mobile application performs real-time object detection based on Mini-YOLOv3, a lightweight version of YOLOv3 object detection, which aims to run on embedded devices such as mobile phones. The proposed mobile application can identify Korean food items based on the input images and displays the food name and ingredient list to the user. The system was tested in a PC environment and it demonstrated a detection accuracy of $68.25 \%$ along with fast testing speed. This study aims to provide future research direction for mobile phone-based real-time object detection.
\end{abstract}

Keywords: Real-time Object Detection, Machine Learning, Korean Food Ingredients, Food Tourism, Mobile Application

\section{Introduction}

Korean food is a significant aspect of the Korean culture that attracts tourists to Korea. Food is also considered to be one of the major factors affecting tourist satisfaction with respect to travel destination because it ensures a more fulfilling traveling experience[1][2]. Studies have found that the local food can enhance the identity of a tourist destination because it is strongly related to the lifestyle, local production, cultural celebration, and heritage of a region[3][4]. According to [5], food tourism can be characterized as tourists visiting the primary and secondary producers of food, food festivals, restaurants, or specific food locations and tasting specialty food items; it is one of the primary motivating factors impacting tourism.

Received(December 23, 2019), Review Result(1st: January 22, 2020, 2nd: March 13, 2020), Accepted(May 27, 2020)

1) (Student) 51140 Dept. of Eco-Friendly Ocean Plant FEED Engineering, Changwon National University, Changwon, Gyeongnam, Korea

email: rifmff@gmail.com

2) (Professor, Corresponding Author) 51140 Dept. of Information and Communication Engineering, Changwon National University, Changwon, Gyeongnam, Korea

email: professorhwang@gmail.com 
Experiencing local food has been found to affect people's behavioral intentions strongly[6]. Therefore, there is a need to address the lack of understanding related to the local food culture[7]. There are crucial situations of food tourism that they able to increase the tension between liking and disliking tendencies in most individuals. Since eating in the unfamiliar environment of the destination can cause disliking tendencies[8].

Moreover, tourists are generally reluctant to consume strange food items whose ingredients are unknown or unfamiliar to them. This may cause some anxiety and a higher risk than most other kinds of contact with the environment[9]. Risks related to food consumption include exposure to unfamiliar ingredients, cooking process and utensils, culinary settings, hygiene, and health risks[10]. Additionally, many tourists are reluctant to eat unfamiliar local food due to concerns about the risk of being unaccustomed to its taste or flavor. Another reason impacting tourist tendencies toward local culinary establishments is the difficulties involved in identifying and ordering the local food items because the tourists are unfamiliar with the names on the menu. Limited knowledge about the local language often aggravates such situations; most of the tourists visiting Korea cannot read the menu or understand the explanations provided by the local staff.

Consequently, most tourists experience difficulties in eating Korean food due to the language barrier or unfamiliarity with the local ingredients[11]. Unfamiliarity tends to create anxiety and disliking tendencies, especially in those tourists that have restrictions related to certain types of ingredients. This may lower the traveling satisfaction. According to [12], six factors that affect the evaluation of the traveling experience, and most of them are related to the importance of familiarity with the ingredients. Therefore, to ensure maximum tourist satisfaction, it is necessary to provide them with information about the local street food ingredients. This research aims to provide this information regarding Korean food to tourists visiting Korea. The remainder of this paper is structured as follows. Section 2 reviews state-of-the-art techniques and research work related to food recognition. Section 3 explains the proposed methodology and architecture. The implementation setup and the achieved results are described in Section 4. Section 5 presents the performance evaluation analysis and discusses the results. Section 6 presents the conclusions and future research direction.

\section{Related Works}

Food recognition is a challenging task because the presentation of food differs even within the same category. Recently, several studies have been conducted on food recognition. Knez et al. 
[13] examined the state-of-the-art food recognition techniques using a mobile device. Sukvichai et al.[14] proposed a method for the classification of food categories and ingredient estimation using Raspberry Pi 3 for Thai cuisine and obtained an accuracy of 80\%; however, the test speed was significantly low. To improve the calculation time, they used PeachPy[15], a Python framework for creating high-performance assembly kernels; consequently, the calculation time was enhanced and the final response time of the system was found to be $3.3 \mathrm{~s}$ per image. Mahmoud et al.[16] proposed the in-depth spectral-spatial features of the snapshot of hyperspectral images for red-meat classification. They are using partial least squares discriminant analysis, support vector machines (SVMs), and convolutional neural networks (CNNs), and obtained an overall accuracy of 95.8\%. The results showed that the 3D-CNN model is much faster than other models. Kawano et al.[17] proposed a real-time food recognition system on a smartphone that could classify 100 different types of food images based on the UEC-FOOD100 dataset using a combination of the HoG patch and a linear SVM; they achieved a classification accuracy of $79.2 \%$ for the top five candidate categories. This study aims to perform real-time detection on Korean food using embedded devices such as .

\section{System Architecture}

\subsection{System Overview}

The primary objective of the proposed system is to provide information related to the ingredients of Korean food items using a mobile application and database service in real-time scenarios. Our research scope is limited to Korean street food. We divided our system features into three main categories, i.e., food detection, food ingredient information, and food information feedback. Users can upload a picture of any Korean food in the proposed mobile application. Then, the application identifies the name of the Korean food using the object detection and machine learning algorithm. It then sends a request to the database server to get a detailed list of ingredients based on the name of the Korean food item. We selected and acquired the images of eight popular Korean street food items, namely teokbokki, odeng, bungeoppang, dakganjeong, gyeranppang, chapssal-donuts, hotteok, and dak-kkochi, for the food image dataset based on the Korea International Foundation on Korean Food Information[18]. We collected 500 images per item. Some images before clipping are shown in [Fig. 1].

After acquiring the image datasets, we performed food detection image training using Mini-YOLOv3 with Python in a PC environment before implementing it in the mobile 

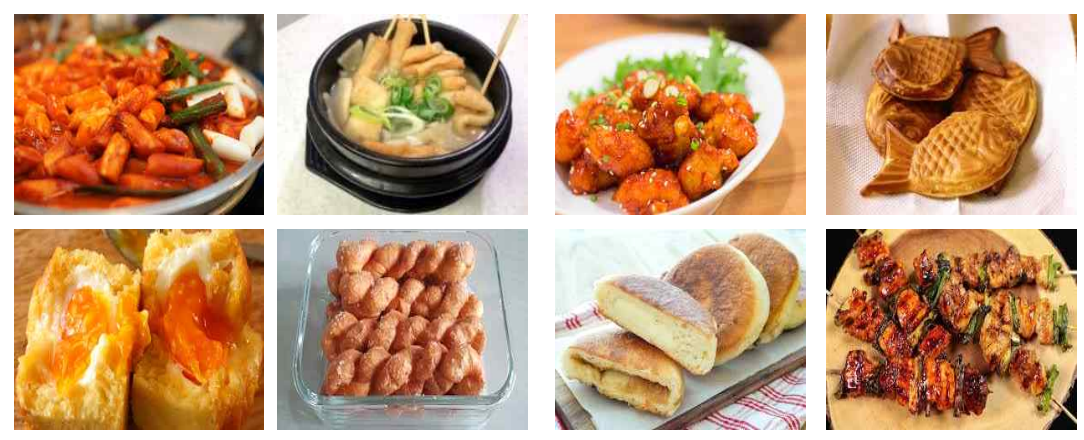

[Fig. 1] Image Dataset Sample

application. We split the image dataset into 400 and 100 images for training and testing, respectively. Subsequently, we converted the food detection code into a software development kit (SDK) with OpenCV4Android to install it in the mobile application. Users can take pictures of Korean street food using the mobile application. The application then performs image detection to identify the name of the Korean street food. When the server receives the name of the food, it finds the detailed ingredient information and sends it to the application. The application retrieves this information and displays it on the user interface. The system architecture and design workflow of the proposed system are illustrated in [Fig. 2] and [Fig. 3], respectively.

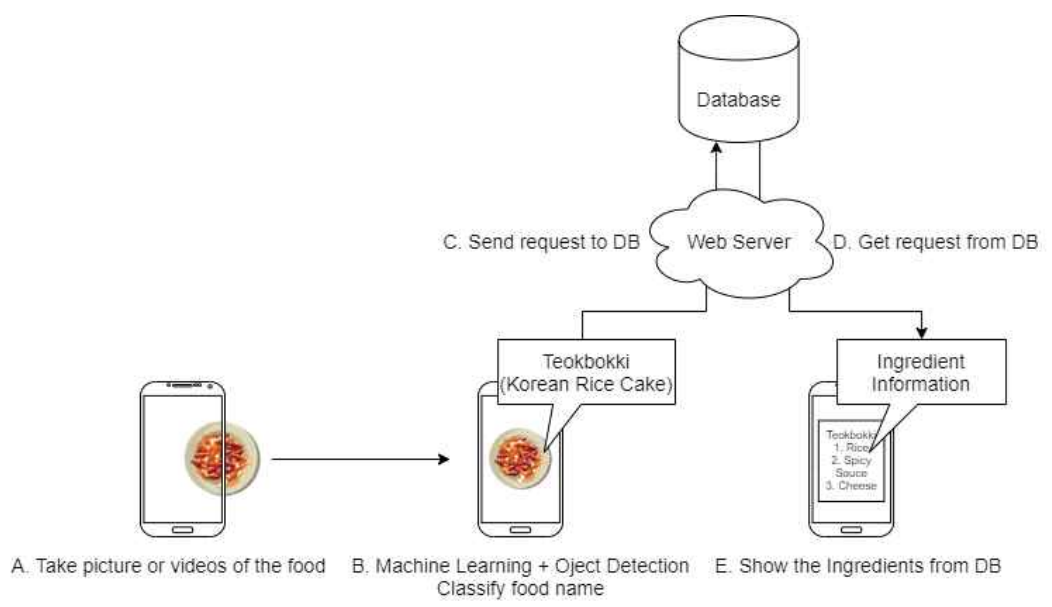

[Fig. 2] System Architecture

First, the user inputs an image of a Korean street food item that they want to classify in the application. Then, the application identifies the name of the input image using the food detection tool. If the input image exists in our database, it is detected correctly, and the system displays the detailed ingredient information. However, if the image is not included in our 
database, the feedback feature is displayed to the users. The feedback feature enables the users to add more food items into our application database.

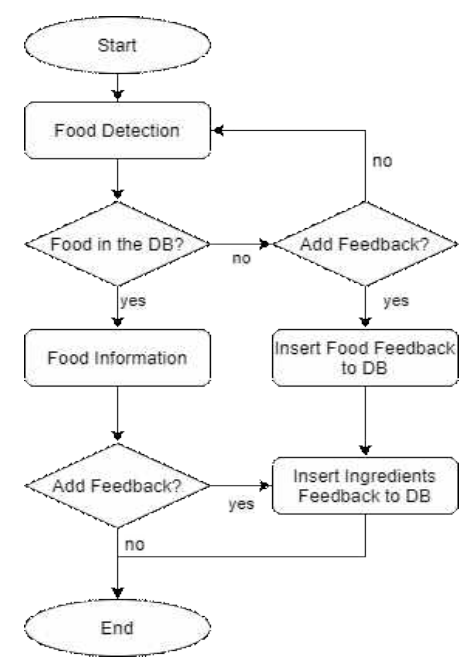

[Fig. 3] Flowchart of the System

\subsection{Food Detection Feature}

In this work, we considered food detection in terms of food localization and recognition. Accordingly, we retrained an object detection algorithm for implementing food detection. Because our system runs on a mobile application in real-time scenarios, we used Mini-YOLOv3 [19], a lightweight network version of YOLOv3[20]. Based on the DarkNet-53 convolutional layer, Mini-YOLOv3 uses separable depth convolutions and pointwise group convolutions to reduce the network's parameter size without particularly reducing the detection accuracy. In comparison to YOLOv3, Mini-YOLOv3 has a smaller model size and fewer trainable parameters and floating-point operations. The parameter size of the feature extraction backbone network is only $16 \%$ of that of DarkNet-53. To compensate for the degradation of accuracy, Mini-YOLOv3 uses a U-shaped, multi-scale feature pyramid network (MSFPN) structure to improve multi-scale object detection performance. Mini-YOLOv3 uses the lightweight backbone network and MSFPN to extract and learn the features from the input image. Then it creates the prediction bounding boxes from the learned features, followed by the non-maximum suppression to generate the desired results. The MSFPN consists of three modules; The concat module combines three feature maps of the backbone network to generate the base feature for constructing the feature pyramid. The encoder-decoder module generates a group of multi-scale features in the form of a U-shaped structure. The feature fusion module combined the three feature maps of the 
backbone network from the concat module and the group of multi-scale features from the encoder-decoder module into a feature pyramid.

\subsection{Food Ingredients Information Feature}

In this research, we used Firebase as the database platform. The Firebase real-time database is a mobile and web application based cloud-hosted NoSQL database that enables users to store and synchronize data across multiple users in real-time. It has three main functions, i.e., storing the user-id, detailed ingredient information, and feedback feature information. Firebase is connected to the Android application using the real-time database feature; additionally, a Java code is incorporated in the mobile application script. The user id database contains personal user information that is necessary for the food information feedback feature.

The mobile application for object detection is developed using Android studio. To implement object detection using a mobile application, we used OpenCV4Android, an open-source computer vision and machine learning software library for Android development. OpenCV4Android works as an SDK library with a set of samples and Javadoc documentation for the OpenCV Java API[21]. In the mobile application, the data sequence starts after the food detection algorithm correctly identifies the Korean street food name. The mobile application sends a request to the database by querying the Korean street food name as a string through the internet to obtain the ingredient information. Then, the database responds with the detailed food ingredient information according to the queried Korean street food name.

\subsection{Food Information Feedback Feature}

This feature is based on whether the picture uploaded by the user does not exist in the Korean food database. Therefore, we added a feature that enables users to upload images of a food item along with its name and ingredients as feedback into our database. Due to this feature, the users can submit their knowledge and experience related to Korean food, thereby increasing the reliability of the application. The feedback feature starts when the application cannot find the relevant food data in the database server. Then, the user is given a choice to either try again or enter a feedback. Users can input food information such as food name, image, and ingredients (optional) using the feedback feature; these data will be saved in the feedback database. In the feedback database, we sorted all feedback inputs based on the names of food items such that the feedback results can be tallied, and created a gallery of user-submitted feedback, as shown in [Fig. 4]. Users can also access and get food information 
from the feedback input gallery.

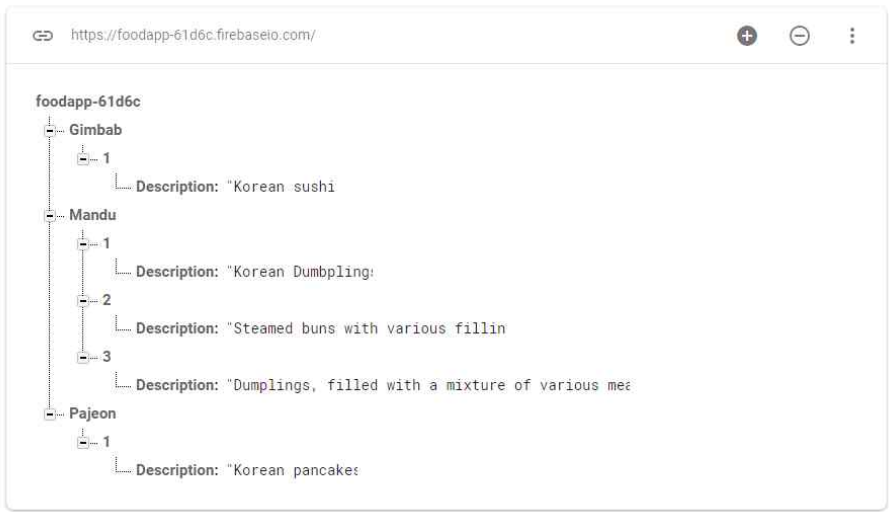

[Fig. 4] Feedback Database

\section{Implementation}

\subsection{Implementation Details}

We developed a lightweight food detection system based on Mini-YOLOv3. The food recognition system was implemented on a mobile application. In our experiments, we used a PC with Intel i5-7500 CPU and NVIDIA GeForce GTX1060 GPU card to train and evaluate the models. For the dataset, we used a Python program based on Microsoft Azure Bing Image Search APIv7. With the Bing Image Search APIv7, we determined specific image search results for eight popular Korean street food items and collected approximately 500 images per item. After creating the dataset, we identified and localized each food item in the form of bounding boxes and cropped the images for further processing. A total of three days were required to train the Mini-YOLOv3 network to learn the entire dataset before the loss function values started overfitting. After training, we tested the training model with the training dataset. For the mobile application, first, we installed and built the OpenCV4Android SDK into our Android studio project. We developed and executed the proposed food detection system on our mobile application emulator, shown in [Fig. 5].

\section{Performance Evaluation}

To evaluate the performance of the proposed system, we calculated the food detection precision of the image tests, which were conducted in a PC environment with Korean street food test 


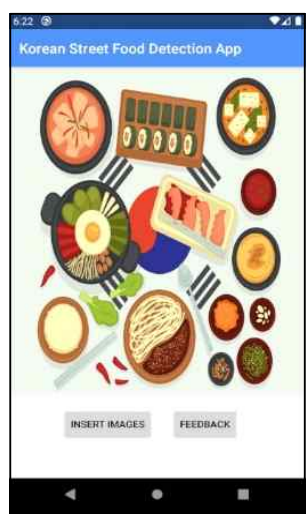

(a)

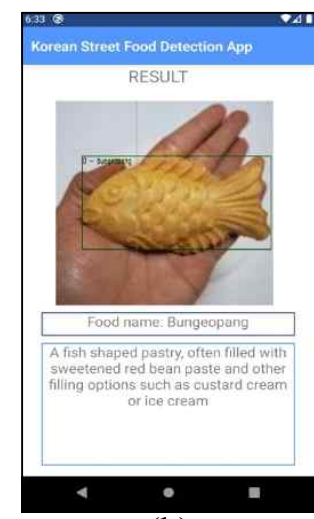

(b)

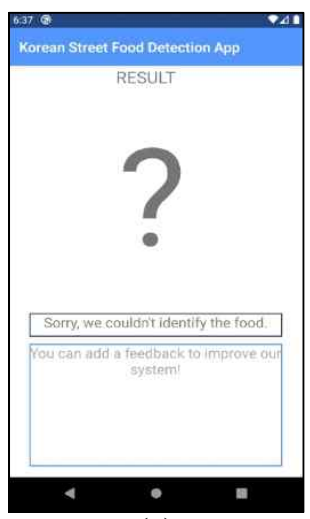

(c)

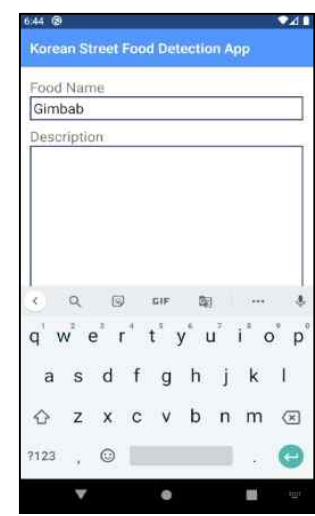

(d)

[Fig. 5] Android Application Interface, (a) Homepage, (b) Food Detection Result, (c) Food not from Database Detection Result, (d) Feedback Feature.

images. We selected 100 images for the test that was different from the training images. The test results showed that the precision of the proposed system should be improved. We obtained an average accuracy of $68.25 \%$ across eight items of the image test dataset, as shown in [Table 2] and [Fig. 6]. The main challenge of this method is misclassification owing to the vast inter-class variability of food items. The detection accuracy of chapssal-donuts was the highest because their presentation does not vary significantly per image. However, the test images for the other food items varied greatly in terms of shape, texture, size, and angle. The detection accuracy for small-sized, non-singular food items such as teokbokki, odeng, and dakganjeong was also observed to be low. Therefore, this system can be improved by optimizing the object segmentation and localization of food objects. The precision of the proposed system is based on a confusion matrix, presented in [Table 1].

[Table 1] Confusion Matrix

\begin{tabular}{|c|c|c|}
\hline & Actual Positive & Actual Negative \\
\hline Predicted Positive & True Positive (TP) & False Positive (FP) \\
\hline Predicted Negative & True Negative (TN) & False Negative (FN) \\
\hline
\end{tabular}

$$
\text { precision }=\frac{T P}{T P+F P}
$$


[Table 2] Precision Table

\begin{tabular}{|c|c|c|c|c|}
\hline Food & Test Image & True & False & Precision \\
\hline Tteokbokki & 100 & 61 & 39 & $61 \%$ \\
\hline Odeng & 100 & 64 & 36 & $64 \%$ \\
\hline Bungeopang & 100 & 71 & 29 & $71 \%$ \\
\hline Dakganjeong & 100 & 65 & 35 & $65 \%$ \\
\hline Gyeranpang & 100 & 70 & 30 & $70 \%$ \\
\hline Chapsal-donuts & 100 & 76 & 24 & $76 \%$ \\
\hline Hotteok & 100 & 72 & 28 & $72 \%$ \\
\hline Dak-kochi & 100 & 66 & 34 & $66 \%$ \\
\hline
\end{tabular}

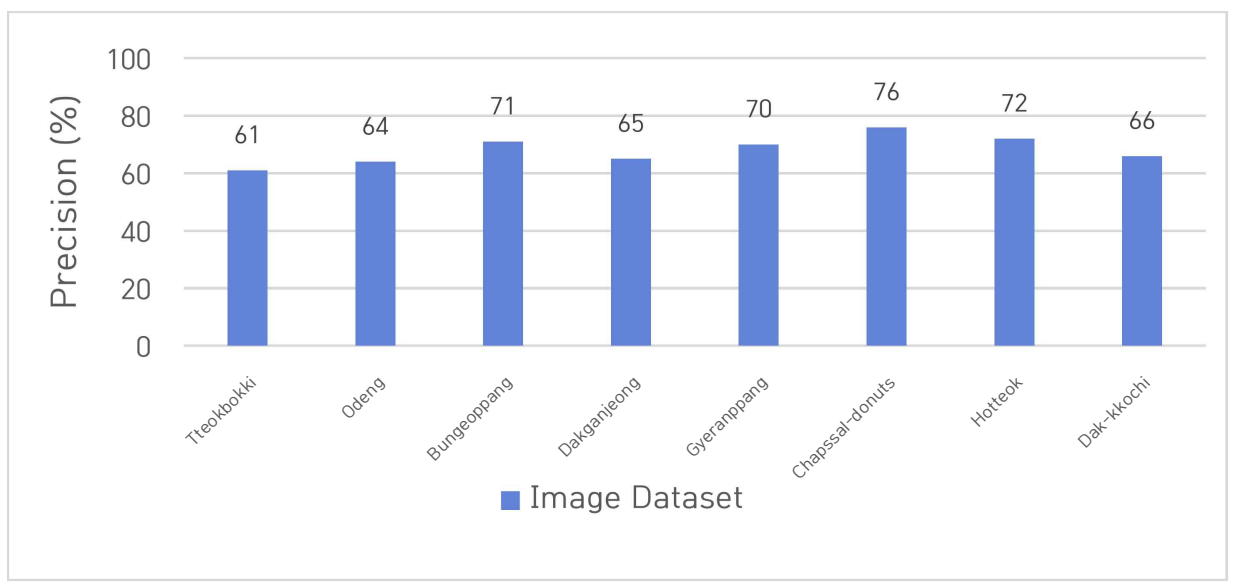

[Fig. 6] Precision Scores

\section{Conclusion}

Limited knowledge of the local food culture can be challenging for tourists because they are mostly unfamiliar with the local language of a region. Therefore, to ensure a pleasant traveling experience, it is essential to provide tourists with information related to the local food. Accordingly, we proposed a mobile application-based food image recognition system to provide information about Korean street food by implementing object detection using the Mini-YOLOv3 network. The system was tested on a PC environment and demonstrated a detection accuracy of $68.25 \%$ along with fast testing speed. However, to improve the system performance, the food object segmentation and localization need to be improved. This study aims to provide future research direction for real-time object detection in embedded devices such as mobile phones. We plan to upgrade our system performance in future by making modifications in the object detection training module and conducting tests based on real food items via field experiments in local Korean markets. We will compare the results of these experiments with those of lab test image experiments. 


\section{Acknowledgement}

This work was supported by Gyeongnam SW Convergence Cluster 2.0 under the contract S0312-20-1002.

\section{References}

[1] S. Yousaf, F. Xiucheng, Halal Culinary and Tourism Marketing Strategies on Government Websites: A Preliminary Analysis, Tourism Management, (2018), Vol.68, pp.423-443, DOI: https://doi.org/10.1016/j.tourman.2018.04.006

[2] C. M. Hall, L. Sharples, The Consumption of Experiences or the Experience of Consumption? An Introduction to the Tourism of Taste, Food Tourism Around the World, UK Oxford: Butterworth-Heinemann Publisher, (2003)

[3] S. Everett, C. Aitchison, The Role of Food Tourism in Sustaining Regional Identity: A Case Study of Cornwall, South West England, Journal of Sustainable Tourism, (2008), Vol.16, No.2, pp.150-167.

[4] C. M. Hall, L. Sharples, R. Mitchell, N. Macionis, B. Cambourne, Food Tourism Around the World, UK Oxford: Butterworth-Heinemann Publisher, (2003)

[5] B. Okumus, F. Okumus, B. McKercher, Incorporating Local and International Cuisines in the Marketing of Tourism Destinations: The Cases of Hong Kong and Turkey, Tourism Management, (2007), Vol.28, No.1, pp.253-261, DOI:10.1016/j.tourman.2005.12.020

[6] R. Sims, Food, Place and Authenticity: Local Food and the Sustainable Tourism Experience, Journal of Sustainable Tourism, (2009), Vol.17, No.3, pp.321-336, https://doi.org/10.1080/09669580802359293

[7] C. T. S. Tsai, Y. C. Wang, Experiential Value in Branding Food Tourism, Journal of Destination Marketing and Management, (2017), Vol.6, No.1, pp.56-65.

[8] J. S. Horng, C. T. S. Tsai, Culinary Tourism Strategic Development: An Asia-Pacific Perspective, International Journal of Tourism Research, (2012), Vol.14, No.1, pp.40-55.

[9] E. Cohen, N. Avieli, Food in tourism - Attraction and Impediment, Annals of Tourism Research, (2004), Vol.31, No.4, pp.755-778.

[10] R. Torres, P. Skillicorn, Montezuma's Revenge: How Sanitation Concerns May Injure Mexico's Tourist Industry, Cornell Hotel and Restaurant Administration Quarterly, (2004), Vol.45, No.2, pp.132-144.

[11] S. Kim, M. Kim, J. Agrusa, A. Lee, Does a Food-themed TV Drama Affect Perceptions of National Image and Intention to Visit a Country? An Empirical Study of Korea TV Drama, Journal of Travel \& Tourism Marketing, (2012), Vol.29, No.4, pp.313-326.

[12] R. C. Y. Chang, J. Kivela, A. H. N. Mak, Attributes that influence the evaluation of travel dining experience: When East meets West, Tourism Management, (2011), Vol.32, No.2, pp.307-316, https://doi.org/10.1016/j.tourman.2010.02.009 
[13] S. Knez, L. Sajn, Food Object Recognition Using a Mobile Device: State of the Art, International Conference on Image Analysis and Processing, (2015), September 7-8; Genoa, Italy

[14] K. Sukvichai, P. Maolanon, K. Sawanyawat, W. Muknumporn, Food Categories Classification and Ingredients Estimation Using CNNs on Raspberry Pi 3, The 10th International Conference on Information and Communication Technology for Embedded Systems, (2019), March 25-27; Bangkok, Thailand

[15] PeachPy, https://github.com/Maratyszcza/PeachPy, Oct 08 (2017)

[16] M. Al-Sarayreh, M. Reis, W. Yan, Deep Spectral-spatial Features of Snapshot Hyperspectral Images for Red-meat Classification, International Conference Image and Vision Computing New Zealand, (2018), November 19-21; Auckland, New Zealand

[17] Y. Kawano, K. Yanai, FoodCam: A Real-time Food Recognition System on a Smartphone, Multimedia Tools and Applications, (2015), Vol.74, No.14, pp.5263-5287.

[18] Korean Food Information, https://www.data.go.kr/dataset/15002968/fileData.do?lang=en, Dec 27 (2018)

[19] J. Redmon, A. Farhadi, YOLOv3: An Incremental Improvement, arXiv:1804.02767, (2018)

[20] Q. C. Mao, H.. M. Sun, Y. B. Liu, R.. S. Jia, Mini-YOLOv3: Real-Time Object Detector for Embedded Applications, IEEE Access, (2019), Vol.7, No.1, pp.133529-133538.

[21] OpenCV for Android, https://opencv.org/android/, Dec 31 (2019) 